\title{
Graduação em Saúde Coletiva no Brasil: Formação, identidade profissional e inserção no mercado de trabalho
}

\author{
Graduation in Public Health in Brazil: Training, professional identity and insertion in the labor \\ market
}

Graduación en Salud Pública en Brasil: Formación, identidad profesional e inserción en el mercado laboral

\author{
Jorgiana de Oliveira Mangueira \\ ORCID: https://orcid.org/0000-0001-7429-3473 \\ Universidade Federal de Pernambuco, Brasil \\ e-mail: jorgiana.mangueira@ufpe.br \\ Luís Roberto da Silva \\ ORCID: https://orcid.org/0000-0002-6194-7615 \\ Universidade Federal de Pernambuco, Brasil \\ e-mail: luis.robertos@ufpe.br \\ Kátia Rejane de Medeiros \\ ORCID: https://orcid.org/0000-0002-7518-4137 \\ Instituto Aggeu Magalhães, Brasil \\ Fundação Oswaldo Cruz, Brasil \\ e-mail: kmedeiros@cpqam.fiocruz.br \\ Carlos Renato dos Santos \\ ORCID: https://orcid.org/0000-0001-8367-7006 \\ Universidade Federal de Pernambuco, Brasil \\ e-mail: carlos.santos@ufpe.br \\ Isabela Cardoso de Matos Pinto \\ ORCID: https://orcid.org/0000-0002-1636-2909 \\ Universidade Federal da Bahia, Brasil \\ e-mail: isabelacmp@gmail.com
}

\begin{abstract}
Resumo
Introdução: Os Cursos de Graduação em Saúde Coletiva (CGSC) surgiram com o intuito de diminuir o tempo de formação e suprir a necessidade de profissionais aptos a atuarem no campo da saúde coletiva no âmbito do Sistema Único de Saúde (SUS). Objetivo: Analisar as publicações sobre os CGSC no Brasil dos últimos anos e caracterizá-las quanto aos principais enfoques estudados. Metodologia: Trata-se de uma revisão integrativa de literatura. A busca de artigos ocorreu nas seguintes bases de dados: Scielo, Lilacs e Scopus. Os descritores saúde coletiva, saúde pública e gestão em saúde foram relacionados com os descritores graduação e sanitarista. Foram incluídos artigos originais, disponíveis na íntegra, relacionados ao objetivo do estudo, publicados no período de 2015 a 2020 no Brasil. A busca relacionou 3.315 artigos, no entanto, apenas 10 artigos atenderam aos critérios de inclusão. Resultados/discussão: Os estudos selecionados foram agrupados em: 1. Panorama, formação e identidade profissional; 2. Inserção no mercado de trabalho. Os cursos apresentam um perfil de formação para a gestão de serviços e sistemas de saúde. A inserção no mercado de trabalho tem se dado de forma gradual, em torno de $43 \%$ de egressos inseridos, a maioria nos serviços públicos ocupando cargos de gerenciamento e gestão de serviços e com vínculos empregatícios precários. Considerações finais: $\mathrm{O}$ estudo foi precursor na análise dos artigos originais, produzidos sobre os CGSC no Brasil permitiu a sumarização das pesquisas realizadas e trouxe um panorama atual sobre o tema da identidade e a inserção desses profissionais no mercado de trabalho.
\end{abstract}

Palavras-chave: Saúde coletiva; Saúde pública; Gestão em saúde; Formação profissional; Sanitarista.

\footnotetext{
Abstract

Introduction: The Undergraduate Collective Health Courses (CGSC) emerged with the aim of reducing training time and meeting the need for professionals able to work in the field of public health within the scope of the Unified Health System (SUS). Objective: To analyze publications on CGSC in Brazil in recent years and to characterize them in
} 
terms of the main approaches studied. Methodology: This is an integrative literature review. The search for articles occurred in the following databases: Scielo, Lilacs and Scopus. The descriptors collective health, public health and health management were related to the descriptors undergraduate and health. Original articles, available in full, related to the objective of the study, published in the period from 2015 to 2020 in Brazil, were included. The search listed 3,315 articles, however, only 10 articles met the inclusion criteria. Results / discussion: The selected studies were grouped into: 1. Panorama, training and professional identity; 2. Insertion in the job market. The courses present a training profile for the management of health services and systems. The insertion in the job market has been done gradually, around $43 \%$ of graduates, most of them in public services occupying management and service management positions and with precarious employment ties. Final considerations: The study was a precursor in the analysis of the original articles, produced on the CGSC in Brazil, allowed the summarization of the researches carried out and brought a current panorama on the theme about the identity and the insertion of these professionals in the job market.

Keywords: Collective health; Public health; Health management; Professional training; Sanitary.

\section{Resumen}

Introducción: Los Cursos de Pregrado en Salud Colectiva (CGSC) surgieron con el objetivo de reducir el tiempo de formación y atender la necesidad de profesionales capaces de trabajar en el campo de la salud pública en el ámbito del Sistema Único de Salud (SUS). Objetivo: Analizar las publicaciones sobre CGSC en Brasil en los últimos años y caracterizarlas en función de los principales enfoques estudiados. Metodología: Se trata de una revisión integradora de la literatura. La búsqueda de artículos se realizó en las siguientes bases de datos: Scielo, Lilacs y Scopus. Los descriptores salud colectiva, salud pública y gestión sanitaria se relacionaron con los descriptores pregrado y salud. Se incluyeron artículos originales, disponibles en su totalidad, relacionados con el objetivo del estudio, publicados en el período de 2015 a 2020 en Brasil. La búsqueda enumeró 3.315 artículos, sin embargo, solo 10 artículos cumplieron con los criterios de inclusión. Resultados / discusión: Los estudios seleccionados se agruparon en: 1. Panorama, formación e identidad profesional; 2. Inserción en el mercado laboral. Los cursos presentan un perfil formativo para la gestión de servicios y sistemas de salud. La inserción en el mercado laboral se ha realizado de forma paulatina, en torno al $43 \%$ de los egresados, la mayoría de ellos en servicios públicos ocupando puestos de dirección y dirección de servicios y con precariedad laboral. Consideraciones finales: El estudio fue precursor en el análisis de los artículos originales, producidos sobre la CGSC en Brasil, permitió resumir las investigaciones realizadas y trajo un panorama actual sobre el tema de la identidad y la inserción de estos profesionales en el mercado laboral.

Palabras clave: Salud colectivo; Salud pública; Gestión en salud; Capacitación profesional; Sanitario.

\section{Introdução}

Em meados da década de 1970 no Brasil, a Saúde Coletiva consolidou-se como campo de conhecimento com múltiplas influências, num período pela oposição ao governo militar e consequente luta pela democratização e pelo pensamento crítico em saúde, contrário às políticas autoritárias e privatizantes de saúde (Baptista, Azevedo \& Machado, 2015; Osmo \& Schraiber, 2015). A expressão Saúde Coletiva surgiu como um substantivo relativamente neutro, a partir da crítica ao modelo médico hegemônico, aos movimentos ideológicos relacionados à medicina social, saúde pública, higiene e medicina preventiva, também permitia a construção de um espaço social não exclusivamente médico (Paim, 2006; Vieira-da-Silva, 2018).

A luta contra a ditadura, a prática política voltada para mudanças das relações sociais, incluiu no projeto da Saúde Coletiva a Reforma Sanitária Brasileira (RSB). Este movimento envolveu estudantes, professores universitários, profissionais de saúde e setores populares que tinham como bandeira principal a luta pela democratização da saúde, que culminou com a origem do Sistema Único de Saúde (SUS) (Paim, 2007; Osmo \& Schraiber, 2015).

No período pós RSB com a criação do SUS houve uma considerável diversificação de cursos de especialização lato sensu, com a assimilação de conhecimentos relacionados às subáreas relacionadas à Saúde Coletiva. A formação em Saúde Coletiva consolidou-se enquanto pós-graduação, nos cursos de especialização em saúde pública, residências de medicina preventiva e social e nos programas de pós-graduação de mestrado e doutorado. Esses cursos foram importantes para a formação de docentes e gestores para a atuação nos diversos âmbitos dos serviços de saúde (Vieira-da-Silva, 2018; Nunes, Fagundes \& Soares, 2015).

Posteriormente, a criação dos Cursos de Graduação em Saúde Coletiva (CGSC) se deu a partir da necessidade de se formar profissionais aptos a atuarem no campo da saúde coletiva para suprir demandas do Sistema Único de Saúde (SUS) e 
diminuir o tempo de formação, uma vez que, antes era necessário realizar uma graduação em alguma área da saúde para, só depois, através da pós-graduação, tornar-se sanitarista (Paim \& Pinto, 2013). Assim, com a criação dos CGSC, foi possível formar profissionais em tempo hábil e voltados a atuar nesse campo através da incorporação do conjunto de saberes e práticas do fazer em saúde coletiva já na graduação (Gerhardt, 2019).

Ademais, o debate para criação dos CGSC foi algo repleto de controvérsias devido à existência da especialização, uma vez que passaram a se formar profissionais específicos para atuar no campo da saúde coletiva e com isso, a inserção de uma nova geração de sanitaristas no mercado de trabalho, que coexiste com àqueles oriundos de outras graduações em saúde, mas com pós-graduação em saúde coletiva/pública e afins (Viana, 2017). Com isso, o movimento pela consolidação do espaço de trabalho e do fazer dos sanitaristas bacharéis ainda é recente e com desafios a serem enfrentados para o reconhecimento da profissão e consolidação de seus espaços de trabalho (Cezar, Ricalde, Santos \& Rocha, 2015).

Por conseguinte, depois de diversas discussões acerca da criação dos CGSC, com a publicação do Decreto no 6.096, de 24 de abril de 2007 que instituiu o Programa de Reestruturação e Expansão das Universidades Federais (REUNI) que tinha o objetivo de proporcionar às universidades federais as condições necessárias para ampliar a oferta, acesso e permanência no ensino superior, os adeptos a criação do curso tiveram a possibilidade de darem os passos iniciais para essa criação em algumas instituições de ensino superior (IES) públicas e passaram a ofertar a graduação em saúde coletiva que foi se expandindo ao longo dos anos pelo país (Lorena et al., 2016).

Após o movimento pela criação dos CGSS e com a formação das primeiras turmas de sanitaristas bacharéis, veio a necessidade de se lutar pelo reconhecimento da profissão do sanitarista formado na graduação, consolidar seu escopo de práticas e saberes e conquistar espaços no mercado de trabalho (Cezar et al., 2015). Atualmente, no Brasil, existem em torno de 22 IES que ofertam os CGSC, saúde pública e outras denominações afins, distribuídos no país (Ministério da Educação, 2020).

Além disso, a luta pelo reconhecimento da profissão é constante e tem mobilizado diversos estudantes e egressos dos CGSC nesse processo de construção da identidade profissional e consolidação dos espaços de trabalho. Mesmo diante da ampla possibilidade de locais de atuação, muitos profissionais ainda não ocupam espaços como estes, devido aos entraves da não consolidação dessa categoria. A não inclusão/reconhecimento dos bacharéis em editais de concursos e seleções para cargos na área da saúde é um fator limitante, que demanda articulação política entre os bacharéis, as Instituições de Ensino Superior e a gestão dos serviços de saúde. Em muitos casos, os cargos de sanitaristas são restritos aos profissionais com outras formações em na área da saúde, com especialização em saúde coletiva/ pública ou por questões políticas (Cezar et al., 2015).

A realização desse estudo justifica-se devido à necessidade de se ampliar o escopo teórico de produções acerca da temática da formação e atuação dos sanitaristas bacharéis e de contribuir com a construção da identidade do profissional sanitarista formado nos CGSC e afins, além de compreender o que abordam as literaturas já publicadas sobre o assunto. Visto isso, esse estudo tem por objetivo analisar as publicações a respeito dos cursos de graduação em saúde coletiva no Brasil, no período de 2015 a 2020 e caracterizá-las quanto aos principais enfoques estudados.

\section{Metodologia}

Este estudo trata-se de uma revisão integrativa de literatura, que tem a finalidade de reunir e sintetizar resultados de pesquisas sobre um tema ou questão delimitada. Este método permite a síntese de múltiplos estudos, possibilitando conclusões gerais a respeito de determinada área de conhecimento (Mendes, Silveira \& Galvão, 2008).

Esta pesquisa buscou analisar as publicações a respeito dos CGSC no Brasil entre os anos de 2015 e 2020 e caracterizá-las quanto aos principais enfoques estudados. A partir do objetivo proposto, foram estabelecidas palavras-chave para busca de artigos nas bases de dados. Os descritores disponíveis no Decs (Descritores em Ciências da Saúde) foram: saúde coletiva, saúde pública e gestão em saúde. Estes foram relacionados com: graduação e sanitarista (não disponíveis no Decs, 
no entanto a sua utilização ampliou o escopo de artigos para análise, por estarem relacionados ao objetivo da pesquisa). A busca de artigos ocorreu durante os meses de março a dezembro de 2020. As bases de dados pesquisadas foram: Scielo (Scientific Electronic Library On-line), Lilacs (Literatura Latino-Americana e do Caribe em Ciências da Saúde) e Scopus.

Os critérios de inclusão definidos foram: artigos originais, frutos de pesquisas documentais ou de campo, disponíveis na íntegra, artigos relacionados ao objetivo do estudo com foco na graduação em saúde coletiva/ pública ou gestão de serviços de saúde, publicações dos últimos anos (2015 a 2020) realizadas no Brasil. Os critérios de exclusão foram: artigos que fugiram ao objetivo da pesquisa, artigos indisponíveis na íntegra, relatos de experiência, revisões de literatura e ensaios teóricos.

A busca nas bases de dados resultou inicialmente num total de 3.315 artigos, sendo 388 encontrados na Scielo, 810 na

Lilacs e 2.117 na Scopus. A análise dos artigos iniciou pela leitura dos títulos dos artigos resultantes das buscas pelos descritores. Foram identificadas 29 publicações relacionadas à graduação em Saúde Coletiva. Após a leitura dos textos na íntegra, aplicados os critérios de inclusão e exclusão, foram incluídos 10 artigos para a análise (Figura 1).

Figura 1: Fluxograma da seleção de artigos para o estudo.

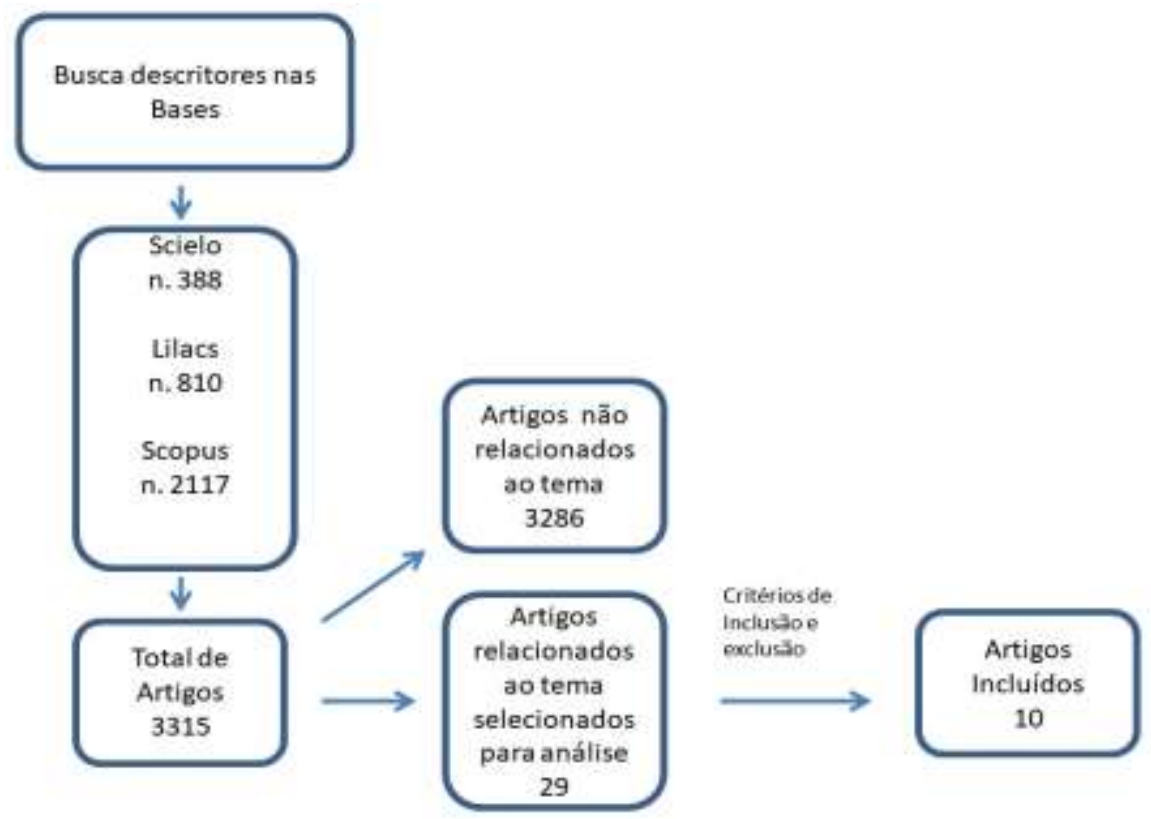

Fonte: Elaborado pelos pesquisadores.

Para a análise dos artigos selecionados, foram elaborados dois formulários para coleta de informações. No primeiro formulário foram extraídas as seguintes informações: título do artigo, ano de publicação, tipo de estudo, objetivo do estudo, revista e base de dados. Foi elaborado um segundo formulário, no qual foram registradas as seguintes informações: título do artigo, população e amostra, local do estudo e principais resultados. Após a coleta dessas informações os resultados foram descritos com base nos principais enfoques estudados. Constatou-se que os principais temas abordados foram: estudo sobre o panorama dos cursos no Brasil, composição curricular, identidade do sanitarista e o processo de inserção no mercado de trabalho. Os resultados encontrados foram analisados descritivamente, discutidos e relacionados com a literatura.

Este estudo utilizou dados secundários, obtidos através de publicações em meios públicos, disponíveis em plataformas digitais, o que prescinde da aprovação em Comitê de Ética, de acordo com a Resolução n. 466, de 12 de dezembro de 2012, do Conselho Nacional de Saúde. 


\section{Resultados e Discussão}

Os artigos selecionados foram dispostos em um quadro e descritos quanto aos seguintes aspectos: título, revista, ano da publicação, tipo de estudo, objetivo do artigo e base de dados (Quadro 1).

Quadro 1: Artigos incluídos no estudo descritos segundo o título e autores, ano da publicação, tipo de estudo, tema, revista e base de dados.

\begin{tabular}{|c|c|c|c|c|c|c|}
\hline $\mathbf{N}$ & ARTIGO/REVISTA & ANO & $\begin{array}{l}\text { TIPO DE } \\
\text { ESTUDO }\end{array}$ & OBJETIVO & TEMA (S) & $\begin{array}{l}\text { BASE DE } \\
\text { DADOS }\end{array}$ \\
\hline 1 & $\begin{array}{l}\text { A área de política, planejamento e } \\
\text { gestão em saúde nas graduações em } \\
\text { saúde coletiva no Brasil. SOBRAL, } \\
\text { Lorena Franco; BARROS, Évelin } \\
\text { Lúcia; CARNUT, Leonardo. Trab. } \\
\text { Educ. Saúde }\end{array}$ & 2017 & $\begin{array}{l}\text { Estudo } \\
\text { documental }\end{array}$ & $\begin{array}{l}\text { Caracterizar os componentes } \\
\text { curriculares identificados nos } \\
\text { cursos de graduação em saúde } \\
\text { coletiva relacionados com a área } \\
\text { de PPGS no Brasil. } \\
\text { Compreender sobre como a área } \\
\text { se comporta na graduação. }\end{array}$ & $\begin{array}{l}\text { Formação e } \\
\text { Identidade } \\
\text { Profissional }\end{array}$ & $\begin{array}{l}\text { Scielo } \\
\text { Lilacs }\end{array}$ \\
\hline 2 & $\begin{array}{l}\text { Competências e habilidades nos } \\
\text { currículos da Graduação em Saúde } \\
\text { Coletiva. LOIOLA, Andrey Almeida; } \\
\text { CYRINO, Eliana Goldfarb; } \\
\text { ALEXANDRE, Fabíola Lucy Fronza } \\
\text { Alexandre. Revista Baiana de Saúde } \\
\text { Pública }\end{array}$ & 2017 & $\begin{array}{l}\text { Estudo } \\
\text { documental }\end{array}$ & $\begin{array}{l}\text { Reconhecer as competências e } \\
\text { habilidades } \\
\text { dos bacharelados em saúde } \\
\text { coletiva com base no conteúdo } \\
\text { dos Projetos Pedagógicos de } \\
\text { Cursos de saúde coletiva } \\
\text { vigentes no país. }\end{array}$ & $\begin{array}{l}\text { Formação e } \\
\text { Identidade } \\
\text { Profissional }\end{array}$ & Lilacs \\
\hline 3 & $\begin{array}{l}\text { Gestão de serviços de saúde: analisando } \\
\text { a identidade na graduação. Saúde } \\
\text { debate [online]. FARIA, Mateus } \\
\text { Aparecido de e SILVA, Analise de } \\
\text { Jesus da. Saúde Debate }\end{array}$ & 2016 & $\begin{array}{l}\text { Pesquisa } \\
\text { documental }\end{array}$ & $\begin{array}{l}\text { Encontrar indicações no Projeto } \\
\text { Político-Pedagógico da } \\
\text { graduação em Gestão de } \\
\text { Serviços de Saúde - versões } \\
2008 \text { e } 2013 \text { - sobre quais } \\
\text { caminhos esse curso tem trilhado } \\
\text { e com qual área de } \\
\text { conhecimento mais se relaciona. }\end{array}$ & $\begin{array}{l}\text { Formação e } \\
\text { Identidade } \\
\text { Profissional }\end{array}$ & $\begin{array}{l}\text { Scielo } \\
\text { Lilacs }\end{array}$ \\
\hline 4 & $\begin{array}{l}\text { Graduação em saúde coletiva no Brasil: } \\
\text { onde estão atuando os egressos dessa } \\
\text { formação? LORENA, Allan Gomes de } \\
\text { et al. Saúde soc. [online]. Saúde } \\
\text { Sociedade. }\end{array}$ & 2016 & $\begin{array}{l}\text { Estudo } \\
\text { descritivo } \\
\text { Abordagem } \\
\text { quantitativa }\end{array}$ & $\begin{array}{l}\text { Realizar um levantamento } \\
\text { nacional dos egressos da } \\
\text { graduação em saúde coletiva no } \\
\text { Brasil. }\end{array}$ & $\begin{array}{l}\text { Inserção no } \\
\text { Mercado de } \\
\text { Trabalho }\end{array}$ & $\begin{array}{l}\text { Scielo } \\
\text { Lilacs } \\
\text { Scopus }\end{array}$ \\
\hline 5 & $\begin{array}{l}\text { Identidade do sanitarista no Brasil: } \\
\text { percepções de estudantes e egressos de } \\
\text { cursos de graduação em Saúde } \\
\text { Pública/Coletiva. SILVA, Vinício } \\
\text { Oliveira da e PINTO, Isabela Cardoso } \\
\text { de Matos. Interface(Botucatu) [online] }\end{array}$ & 2018 & $\begin{array}{l}\text { Exploratório } \\
\text { Abordagem } \\
\text { qualitativa }\end{array}$ & $\begin{array}{l}\text { Analisar a construção da } \\
\text { identidade profissional dos } \\
\text { estudantes e egressos dos cursos } \\
\text { de graduação em Saúde } \\
\text { Pública/Coletiva no Brasil. }\end{array}$ & $\begin{array}{l}\text { Formação e } \\
\text { Identidade } \\
\text { Profissional }\end{array}$ & $\begin{array}{l}\text { Scielo } \\
\text { Lilacs }\end{array}$ \\
\hline 6 & $\begin{array}{l}\text { Identidade profissional e movimentos } \\
\text { de emprego de egressos dos cursos de } \\
\text { graduação em Saúde Coletiva. Saúde } \\
\text { debate [online]. SILVA, Vinício } \\
\text { Oliveira da; PINTO, Isabela Cardoso de } \\
\text { Matos e TEIXEIRA, Carmen Fontes } \\
\text { de Souza. Saúde e debate. }\end{array}$ & 2018 & $\begin{array}{l}\text { Exploratório. } \\
\text { Abordagem } \\
\text { Qualitativa }\end{array}$ & $\begin{array}{l}\text { Analisar a construção da } \\
\text { identidade profissional dos } \\
\text { estudantes e egressos dos cursos } \\
\text { de graduação em Saúde } \\
\text { Pública/Coletiva no Brasil. }\end{array}$ & $\begin{array}{l}\text { Identidade } \\
\text { Profissional } \\
\text { / Inserção } \\
\text { no Mercado } \\
\text { de Trabalho }\end{array}$ & $\begin{array}{l}\text { Scielo } \\
\text { Lilacs }\end{array}$ \\
\hline 7 & $\begin{array}{l}\text { Interprofissionalidade na Graduação em } \\
\text { Saúde Coletiva: olhares a partir dos } \\
\text { cenários diversificados de } \\
\text { aprendizagem. } \\
\text { PARO, César Augusto; PINHEIRO, } \\
\text { Roseni. Interface (Botucatu) [online]. }\end{array}$ & 2018 & $\begin{array}{l}\text { Exploratório } \\
\text { observacional } \\
\text { Abordagem } \\
\text { qualitativa }\end{array}$ & $\begin{array}{l}\text { Compreender como a } \\
\text { interprofissionalidade é } \\
\text { produzida nos cenários } \\
\text { diversificados de aprendizagem } \\
\text { na formação da graduação em } \\
\text { Saúde Coletiva. }\end{array}$ & $\begin{array}{l}\text { Formação e } \\
\text { Identidade } \\
\text { Profissional }\end{array}$ & $\begin{array}{l}\text { Scielo } \\
\text { Lilacs }\end{array}$ \\
\hline
\end{tabular}




\begin{tabular}{|c|c|c|c|c|c|c|}
\hline 8 & $\begin{array}{l}\text { Mercado de trabalho do sanitarista em } \\
\text { saúde coletiva: novas perspectivas. } \\
\text { SOUZA, Paula Beatriz et al. Revista } \\
\text { Baiana de Saúde Pública }\end{array}$ & 2018 & $\begin{array}{l}\text { Descritivo } \\
\text { utilizando base } \\
\text { dados } \\
\text { secundários, } \\
\text { abordagem } \\
\text { quantitativa }\end{array}$ & $\begin{array}{l}\text { Realizar uma reflexão das } \\
\text { oportunidades de inserção no } \\
\text { mercado de trabalho para o } \\
\text { profissional sanitarista e } \\
\text { entender as esferas de formação } \\
\text { de graduação, mestrado e } \\
\text { doutorado em SC existentes no } \\
\text { Brasil }\end{array}$ & $\begin{array}{l}\text { Inserção no } \\
\text { Mercado de } \\
\text { Trabalho }\end{array}$ & Lilacs \\
\hline 9 & $\begin{array}{l}\text { Os novos sanitaristas no mundo do } \\
\text { trabalho: um estudo com graduados em } \\
\text { saúde coletiva. VIANA, Jussara Lisboa } \\
\text { e SOUZA, Elizabethe Cristina } \\
\text { Fagundes de. Trab educ e saúde } \\
\text { [online]. }\end{array}$ & 2018 & $\begin{array}{l}\text { Exploratório } \\
\text { abordagem } \\
\text { qualitativa }\end{array}$ & $\begin{array}{l}\text { Discorrer sobre a inserção } \\
\text { profissional dos bacharéis em } \\
\text { Saúde Coletiva, especificamente } \\
\text { quanto aos desafios, } \\
\text { potencialidades e estratégias de } \\
\text { inserção no mundo do trabalho. }\end{array}$ & $\begin{array}{l}\text { Inserção no } \\
\text { Mercado de } \\
\text { Trabalho }\end{array}$ & $\begin{array}{l}\text { Scielo } \\
\text { Lilacs }\end{array}$ \\
\hline 10 & $\begin{array}{l}\text { Panorama dos cursos de graduação em } \\
\text { saúde coletiva no Brasil entre } 2008 \text { e } \\
\text { 2014. MENESES, Jéssica Janaina } \\
\text { Santos et al. Trab educ e saúde } \\
\text { [online]. }\end{array}$ & 2017 & $\begin{array}{l}\text { Estudo } \\
\text { documental }\end{array}$ & $\begin{array}{l}\text { Descrever o processo de } \\
\text { expansão dos CGSC no Brasil } \\
\text { entre } 2008 \text { e } 2014 \text { e caracterizar } \\
\text { os } \\
\text { cursos em funcionamento quanto } \\
\text { à distribuição regional e aos } \\
\text { números de vagas ofertadas, } \\
\text { alunos matriculados e bacharéis } \\
\text { graduados. }\end{array}$ & $\begin{array}{l}\text { Panorama, } \\
\text { Formação e } \\
\text { Identidade } \\
\text { Profissional }\end{array}$ & $\begin{array}{l}\text { Scielo } \\
\text { Lilacs }\end{array}$ \\
\hline
\end{tabular}

Fonte: Autores.

Quanto ao ano de publicação, destacamos que no ano de 2016 foram publicados 2 artigos, 3 artigos no ano de 2017 e 5 artigos publicados no ano de 2018. Observou-se que nos anos de 2019 e 2020 não foram encontrados artigos originais sobre os CGSC nas bases de dados pesquisadas. No que se refere ao tipo de estudo, observou-se que 4 artigos selecionados foram estudos documentais envolvendo os Projetos Políticos Pedagógicos dos Cursos (PPP), seguidos por 2 estudos descritivos e exploratórios de abordagem quantitativa e 4 artigos com abordagem qualitativa. A revista Trabalho, Educação e Saúde envolveu a maioria dos estudos selecionados, 3 artigos. Entre as bases pesquisadas, a Lilacs apresentou uma maior amplitude na busca de artigos relacionados ao tema da pesquisa, nesta foram encontrados os 10 artigos selecionados para o estudo. Os mesmos artigos selecionados se repetiram na busca nas demais bases de dados: 8 na Scielo e 1 na Scopus.

Quanto aos objetivos dos estudos selecionados e temas abordados, observou-se que a maioria dos artigos envolveu a discussão sobre o panorama dos cursos no Brasil, a formação e a identidade profissional (7 artigos) e a inserção no mercado de trabalho (4 artigos). Um artigo abordou os dois temas identificados.

\section{Panorama, formação e identidade profissional da graduação em saúde coletiva}

O estudo realizado por Meneses et al. (2017) caracterizou o processo de expansão dos CGSC no Brasil entre 2008 e 2014, a partir da análise dos PPP dos cursos e de um formulário preenchido pelos coordenadores dos cursos, disponíveis na coordenação do Fórum de Graduação em Saúde Coletiva-Abrasco. Os primeiros CGSC no Brasil foram abertos em 2008 na Universidade Federal do Acre (AFAC) e na Universidade de Brasília (UNB) e em 2014 já havia 18 cursos no Brasil, destes, 13 denominavam-se CGSC ou como Graduação em Saúde Pública e 4 cursos denominavam de Gestão em Saúde Coletiva e 17 eram ofertados por universidades públicas (14 em instituições federais e três em instituições estaduais) e um por uma instituição privada (ASCES, Pernambuco).

Em 2014, a região Nordeste sediava a maior parte dos CGSC, 5 (27,8\%), destes, 3 no estado de Pernambuco, 1 no Rio Grande do Norte e 1 na Bahia. Nas demais regiões do país, a maior parte dos estados já possuía pelo menos um CGSC em funcionamento em 2014. Em 2014, os CGSC ofertaram 979 vagas para novos estudantes, número quase seis vezes maior do que o ofertado em 2008. O número de estudantes com matrículas ativas nesses cursos totalizou 2.532 no primeiro semestre de 2014. E até o primeiro semestre de 2014, 285 bacharéis em saúde coletiva já haviam sido titulados (Meneses et al., 2017). 
De acordo com consulta aos dados do e-MEC, atualmente existem 22 IES que ofertam cursos de bacharelado, na modalidade presencial, relacionados à área da saúde coletiva. Quantificar os cursos é uma tarefa delicada, tendo em vista a não homogeneidade quanto à denominação do curso entre as diferentes IES. Constatamos que os cursos são denominados de: graduação em saúde coletiva (16), em saúde pública (1), gestão de serviços de saúde (1), gestão em saúde (1), saúde coletiva indígena (1) e outros 2 cursos de gestão em saúde ambiental. Detectamos que a Universidade de Brasília possui dois cursos com projetos pedagógicos distintos: um da Faculdade de Ceilândia e o outro da Faculdade de Ciências da Saúde perfazendo um total de 22 IES e 23 cursos de graduação (Ministério da Educação, 2020).

Dentre as 22 IES identificadas, 16 são Universidades Federais, 4 Estaduais e 2 Privadas. Quanto à sua distribuição por regiões do país, 6 estão situadas na Região Norte, 5 na Região Nordeste, 5 na Região Sudeste, 4 na Região Sul e 2 na Centrooeste (Ministério da Educação, 2020).

O estudo realizado por Sobral, Barros e Carnut (2017) caracterizou os componentes curriculares de 15 CGSC no Brasil quanto à presença de conteúdos relacionados à gestão de sistemas de saúde, elencados no Termo de Referência para as Diretrizes Curriculares Nacionais (DCN) para os CGSC. Os autores constataram que 97\% dos cursos são ofertados por instituições públicas de ensino e que não possuem padronização quanto ao nome, 60\% denominam-se Saúde Coletiva e 13\% Saúde Pública, Gestão em Saúde, dentre outras denominações. Tais resultados corroboram com os achados sobre os cursos de graduação disponíveis no e-MEC, conforme apresentados anteriormente: não homogeneidade quanto à denominação dos cursos e predomínio da oferta por IES públicas federais (Ministério da Educação, 2020).

Quanto às distintas denominações dos cursos entre Saúde Coletiva, Saúde Pública, Gestão de Sistemas de Saúde, destaca-se que a distinção entre Saúde Pública e Saúde Coletiva não é apenas uma questão de nomes, há noções vinculadas a ideologias e conceitos vinculados a teorias. Ambas expressam em si uma multiplicidade de movimentos, teorias e ressignificações sobre a saúde (Paim \& Pinto, 2013; Lorena \& Akerman, 2016). A expressão Saúde Coletiva é genuinamente brasileira e surgiu como um substantivo relativamente neutro, a partir da crítica ao modelo médico hegemônico, aos movimentos ideológicos relacionados à medicina social, saúde pública, higiene e medicina preventiva, também permitia a construção de um espaço social não exclusivamente médico (Paim, 2006; Vieira-da-Silva, 2018).

O estudo de Sobral et al. (2017) detectou que os conteúdos 'Planejamento em saúde' e 'Política de saúde como política de proteção social' foram citados ao menos uma vez em $60 \%$ e $46 \%$ das matrizes, respectivamente. Não encontrou referência ao SUS e as 'Políticas sociais' em $87 \%$ e 53\% das matrizes analisadas, respectivamente. Observou-se também que $80 \%$ não continham referência à 'Regulação em Saúde' e 'Auditoria em saúde'. O conteúdo sobre 'Administração em saúde' foi contemplado em $80 \%$ e $53 \%$ das matrizes citaram ao menos uma vez 'Monitoramento e avaliação em saúde'. O estudo constatou que a maior parte dos cursos não contempla conteúdos fundamentais da área de Política, Planejamento e Gestão em Saúde, lacunas em conteúdos sobre políticas e da defesa do SUS. Os autores destacam a necessidade de uma padronização curricular, com conteúdos mínimos necessários à formação do sanitarista (Sobral et al., 2017)

Com o intuito de reconhecer as competências e habilidades dos bacharelados em saúde coletiva, foram analisados os conteúdos dos PPP de cursos de saúde coletiva vigentes no país. O estudo de Loiola, Cyrino e Alexandre (2017) foi realizado com cursos de cinco IES de diferentes regiões do Brasil: Universidade Federal do Acre (UFAC), Universidade Federal da Bahia (UFBA), Universidade de Brasília (UNB), campus Ceilândia, Universidade Federal do Rio de Janeiro (UFRJ), Universidade Federal do Rio Grande do Sul (UFRGS).

A análise dos PPP identificou oito núcleos temáticos: Gestão, Política e Planejamento em Saúde (este foi o mais citado entre os projetos pedagógicos analisados); Pesquisa, Inovação e Produção do Conhecimento em Saúde; Cidadania, Participação e Direito à Saúde; Epidemiologia e Vigilância em Saúde; Promoção e Educação em Saúde; Abordagem, Intervenção e Relações Sociais em Saúde; Informação em Saúde; e Auditoria, Controle e Fiscalização de Serviços de Saúde. 
De maneira geral os PPP estudados apresentaram semelhanças quanto às competências e habilidades dos cursos de graduação e consonância com a proposta das DCN para o curso e algumas singularidades específicas em cada um dos cursos analisados. Os autores concluem que é possível compreender o perfil das competências dos novos sanitaristas, que estas apresentam uma identidade bem estabelecida. O estudo ressalta que este resultado pode se dar pela escolha da amostra ser constituída por cursos com a mesma nomenclatura e por envolver cursos criados nos anos de 2008 e 2009. Ressalta que os cursos criados posteriormente podem apresentar divergências quanto a essa identidade (Loiola et al., 2017).

As fragilidades observadas quanto aos conteúdos relacionados à área da gestão em saúde no estudo de Sobral et al. (2017), sinalizam para um aprofundamento e melhor delineamento da identidade do sanitarista graduado. Em contrapartida, os achados de Loiola et al. (2017), já apontam para uma convergência e identidade bem estabelecida, entre os cursos analisados, com vocação para a formação voltada para a gestão de serviços e sistemas de saúde. A formação de recursos humanos capacitados para a atuação no SUS fundamentou a estruturação dos cursos de graduação em Saúde Coletiva. A proposta da graduação justificou-se na necessidade preencher lacunas apontadas pelo mercado de trabalho, no campo da gestão dos serviços e sistemas de saúde, com domínio de conhecimento na epidemiologia, planejamento, programação, avaliação, vigilância em saúde e promoção da saúde (Pinto \& Paim, 2015).

A minuta com as Diretrizes Curriculares Nacionais (DCN) para os cursos de graduação em Saúde Coletiva (2017) estabelece o perfil do bacharel em Saúde Coletiva. Orienta que esta formação envolve os núcleos de conhecimentos e práticas voltados para a Gestão em Saúde, Atenção à Saúde e Educação e comunicação em Saúde. Prevê uma formação generalista, com capacidade para atuar na análise, monitoramento e avaliação em saúde. Formular políticas de saúde, além do planejamento e avaliação de sistemas e serviços de saúde, desenvolvimento de ações intersetoriais de educação e promoção da saúde, além de ações de vigilância, desenvolvimento científico e tecnológico na área de saúde.

Faria e Silva (2016) analisaram duas versões (2008 e 2013) do Projeto Político Pedagógico (PPP) do Curso de Gestão em Serviços de Saúde da UFMG a fim de identificar os caminhos trilhados por esta graduação e qual área de conhecimento o curso mais se relaciona. $\mathrm{O}$ curso apresenta três eixos na formação: área da administração, da saúde coletiva e multidisciplinar. Envolve três unidades acadêmicas da UFMG: enfermagem, Faculdade de ciências econômicas e medicina.

Constatou-se que a área da Administração é central em ambas as versões dos PPP, no entanto a última versão de 2013 já apresenta componentes curriculares com orientação para a área da saúde e uma diminuição do papel da área da administração. Destaca-se que o curso contempla as áreas da formação: ciências humanas e sociais aplicada à saúde, epidemiologia e política, planejamento em saúde, no entanto o curso não é engajado na discussão da formação em saúde. Percebe-se a forte relação entre a saúde coletiva e os conceitos basilares do curso. A vertente multidisciplinar do curso busca transcender as perspectivas do administrador e do sanitarista, bem como identifica fragilidades em ambas as áreas e busca desvincular-se das mesmas, considerando-as insuficientes para a formação do gestor e sistemas de saúde. Os autores identificaram divergências entre os eixos do curso, apontam para a necessidade do diálogo para discussão do processo identitário da referida graduação. Por fim, destacam que a vertente da saúde coletiva é amparada por produções acadêmicas, organizações da sociedade e um maior apoio nacional (Faria \& Silva, 2016).

A análise realizada nos PPP do Curso de Gestão de Serviços de Saúde da UFMG por Faria e Silva (2016), demonstra um claro conflito e indefinição da identidade do curso, que nasceu mais vinculado à área da Administração, com tendência maior na segunda versão do PPP para a área da saúde. A tendência do curso em optar pela vertente multidisciplinar é destacada pelos autores como algo a se refletir e estreitar diálogos a fim de consolidar a identidade da referida graduação. Os autores reconhecem os avanços da vertente da saúde coletiva, que vem se consolidando com a formação de profissionais voltados para a gestão dos serviços de saúde. Outros cursos, antes denominados Gestão de Serviços de Saúde, como o da UFRN, ao passar pelo processo de reforma curricular, se reconheceram como graduação em saúde coletiva (Viana, 2017). 
Paro e Pinheiro (2018) pesquisaram como a interprofissionalidade é produzida nos cenários de aprendizagem na formação da graduação em Saúde Coletiva. O estudo foi realizado com 15 discentes do curso de Saúde Coletiva da Universidade Federal do Rio de Janeiro (UFRJ). Constatou-se que as práticas interprofissionais nos cenários de aprendizagem apresentaram dificuldade de manter a integralidade das atividades, principalmente naquelas que tinha enfoque interventivo e exigia colaboração com os demais atores das gestões e serviços de saúde.

As interações com os diferentes profissionais da gestão, serviços e de outros setores foram destacados como pontos positivos para a formação, por ser um reflexo do cotidiano dos serviços. Essa interação também possibilitou a união dos saberes e práticas e, consequentemente, colaborando para a efetivação da interprofissionalidade nos serviços de saúde. Os entrevistados destacaram ainda que muitas vezes se sentiam parte integrante das equipes multiprofissionais dos serviços e apresentavam satisfação com a atuação conjunta de profissionais e estudantes, fortalecendo, assim, o vínculo entre eles. Também foram relatadas dificuldades em trabalhar em equipe, integração de alguns profissionais para colaboração nas atividades acadêmicas dos estudantes, a baixa articulação entre universidade e serviços, falta de acompanhamento da tutoria nos campos de prática e o despreparo de alguns professores frente à formação (Paro \& Pinheiro, 2018).

O tema da interprofissionalidade tem mobilizado desde educadores, gestores a organismos e instituições de saúde e educação. No entanto, ainda estão presentes muitas contradições quanto aos limites e as resistências políticas, teóricas e práticas presentes na educação interprofissional. $\mathrm{O}$ debate sobre a interprofissionalidade ainda é muito ausente no cotidiano da graduação em saúde, no entanto algumas experiências como na Universidade Federal do Sul da Bahia (UFSB) indicam que o exercício da formação em saúde em articulação com o SUS tem possibilitado o surgimento de novos arranjos em relação ao modelo biomédico de profissionalização (Pereira, 2018).

O estudo de Silva e Pinto (2018) analisou a identidade do sanitarista graduado em saúde coletiva. A pesquisa foi realizada com 16 egressos e estudantes que participaram do VII Reunião do Fórum Nacional de Graduação em Saúde Coletiva. Constatou-se que, embora a maioria dos entrevistados não tivesse escolhido o CGSC como primeira opção, os mesmos após ingressarem despertaram o pertencimento a esse campo. No tocante à formação, viu-se que embora os cursos tenham maior foco na gestão dos serviços de saúde, denota-se a variedade de espaços de práticas para os sanitaristas, ampliando a inserção em espaços governamentais ou não governamentais. O compromisso de formação político-social dos indivíduos para que eles sejam capazes de mudar a realidade dos serviços de saúde do país, a partir do compromisso com a transformação dos serviços de saúde do país e dos princípios da reforma sanitária brasileira também foi destacado pelos entrevistados.

As DCN dos cursos de graduação em Saúde Coletiva consideram como cenários de aprendizagem as redes de gestão e atenção em saúde, orienta a inserção dos graduandos nestes espaços desde o início do curso. Também orienta a criação de oportunidades integradas de aprendizagem ao longo do curso, "tendo a Epidemiologia, a Política, o Planejamento e Gestão em Saúde e as Ciências Sociais e Humanas em Saúde como eixos fundamentais da formação" (p. 6). Os cenários de prática apontados para os egressos dos cursos de graduação envolvem: hospitais públicos ou privados, centros e unidades de saúde, policlínicas, unidades de urgência e emergência, saúde suplementar, organizações sociais, secretarias estaduais e municipais e ministério da saúde (Pinto \& Paim, 2015).

Silva, Pinto e Teixeira (2018) ao estudarem os aspectos relacionados à identidade profissional dos estudantes e egressos dos CGSC, destacam que o reconhecimento do trabalho colabora para a construção da identidade pessoal e criatividade social, que a privação do trabalho implica em sofrimento para o indivíduo. O estudo aponta dificuldades a serem superadas na construção da identidade profissional do sanitarista. Considerando que esse profissional tem sido formado para atuar, predominantemente, no setor público, a construção da sua identidade possui estreita relação com as políticas de saúde e o processo de construção do SUS no Brasil.

Corroborando com tais achados sobre a construção da identidade profissional, Silva e Pinto (2013) afirmam que esta 
se constitui a partir de sucessivas socializações, sendo permanentemente reconstruída. Revela o sentimento de pertencimento a um determinado grupo. A mesma tem sido utilizada para compreender o processo de inserção dos sujeitos no mercado de trabalho. O processo de definição da identidade profissional perpassa pela interseção de três fatores: o mundo vivido do trabalho, a trajetória socioprofissional e os movimentos de emprego. As crises de identidade profissional muitas vezes estão relacionadas às dificuldades de inserção profissional, exclusão social, desagregação da categoria, constantes mudanças no mercado de trabalho (Silva \& Pinto, 2013).

Para Bosi e Paim (2010), os graduados deverão ajudar na configuração mais clara de sua identidade, uma vez que, os elementos que conferem identidade própria ao profissional são elaborados a partir de um processo de socialização.

\section{Inserção no Mercado de Trabalho}

Dentre os estudos analisados, observou-se que 4 artigos abordaram a inserção dos sanitaristas bacharéis no mercado de trabalho. O estudo de Lorena et al. (2016) realizou um levantamento nacional dos egressos de saúde coletiva no Brasil. Foi apontado que, dos 144 egressos do CGSC do Brasil, 42,4\% estavam atuando como sanitaristas no mercado de trabalho. Quanto aos locais de trabalho, esses egressos estavam atuando em secretarias municipais de saúde, instituições de pesquisa, Ministério da Saúde, secretarias estaduais de saúde, hospitais municipais e hospitais sob gestão de Organização Social de Saúde (OSS) e setor privado.

O Mercado de trabalho para as novas profissões surge paralelamente ao desenvolvimento dos cursos, desta forma, ainda está em construção, constitui-se num processo dinâmico, que requer tempo (Lorena \& Akerman, 2016). O Sanitarista graduado, por ser uma ocupação nova, pode encontrar algumas barreiras para inserção no mercado de trabalho, pelo desconhecimento por parte de gestores do sistema.

Viana e Souza (2018) analisaram os desafios, potencialidades e estratégias de inserção do sanitarista no mundo do trabalho junto a 37 egressos do curso de saúde coletiva da UFRN. A dificuldade de reconhecimento do sanitarista graduado no mercado de trabalho foi apontada como um dos principais obstáculos para efetivação do ingresso. Apontaram que aqueles que já ocupam espaços no mercado de trabalho, passam por uma instabilidade empregatícia pelo fato dos cargos serem comissionados ou de contrato temporário, com vínculos precários e acabam por sofrerem com interferências políticas.

Pochmann (2020), em estudo realizado sobre tendência do mundo do trabalho no Brasil, incluiu na categoria precarização a soma das ocupações de assalariamento informal, os que trabalhavam por conta própria e as pessoas sem remuneração. A precarização caracteriza-se pela adoção de formas de contratação temporária, sem estabilidade, sem registro em carteira, tanto em atividades instáveis, temporárias ou na situação de desemprego. Estamos diante das modalidades de precarização, na qual a terceirização tem se destacado. Em muitas empresas/instituições o número de trabalhadores terceirizados supera o número de trabalhadores efetivos (Antunes, 2018).

O estudo de Silva et al. (2018) analisou a construção da identidade profissional dos egressos dos CGSC no Brasil, através de um grupo focal com 16 estudantes. O estudo também possui achados comuns aos apontados anteriormente, mas outro ponto interessante para discussão é o conflito entre sanitaristas formados nos CGSC e aqueles oriundos da pósgraduação, que já atuam nos serviços de saúde. O estudo aponta que ainda há uma determinada resistência desses profissionais em receberem os bacharéis em saúde coletiva. Destaca que a articulação das IES com os serviços de saúde facilita a eventual inserção no mercado de trabalho, a precariedade dos vínculos e o setor público com o principal campo de atuação, também foram apontados. Evidencia-se um descompasso entre a necessidade desse novo perfil profissional e sua inserção no mundo do trabalho. Tal fato aponta para a necessidade de mobilização dos estudantes, egressos, professores, gestores e outros sujeitos comprometidos com a manutenção e o fortalecimento do SUS para fortalecer essa identidade profissional. 
Estes novos sanitaristas graduados se encontram em meio a disputas no mercado de trabalho, em relação aos profissionais das outras áreas da saúde, como os profissionais pós-graduados (Lato ou Stricto sensu) na área da saúde coletiva, que historicamente vem ocupando os espaços de gestão após o investimento na sua formação (Ianni, 2016).

Outro apontamento importante referente às dificuldades de inserção no mercado de trabalho é a baixa remuneração desses profissionais. Alguns aceitam a oportunidade por não terem condições socioeconômicas para esperar por outras com melhores remunerações e/ou por acreditarem que a experiência profissional contribui para melhor consolidação no mercado de trabalho. A dedicação exclusiva aos programas de pós-graduação e/ou aos empregos anteriores à graduação também são motivos pelos quais os egressos dos CGSC não estejam inseridos no mercado de trabalho como sanitaristas, como apontado por Lorena et al. (2016) e Silva et al. (2018).

Ademais, quanto às potencialidades da inserção no mercado de trabalho, Viana e Souza (2018), apontaram que a inserção dos graduandos nos serviços de saúde por meio das atividades práticas e estágios curriculares ou extras, são fortes oportunidades para consolidação da profissão de bacharéis em saúde coletiva nos serviços de saúde, pois permite aos profissionais atuantes que conheçam a atuação desses sanitaristas e também permite a consolidação dos espaços de trabalho desse público.

Outro ponto que se destaca é a amplitude de possibilidades de atuação desses profissionais sanitaristas, Lorena et al. (2016), Viana e Souza (2018) e Souza, Lopes, Valença e Medeiros (2018) apontam algumas dessas possibilidades, dentre elas estão: coordenação de unidades de saúde, de pesquisas e políticas públicas; sanitaristas do Núcleo Ampliado de Saúde da Família e Atenção Básica (NASF-AB); formação docente; consultoria técnica; analistas de políticas públicas e sistemas de saúde; programas governamentais do Ministério da Saúde; gerência de serviços de atenção básica, média e alta complexidade hospitalar e ambulatorial; atividades e/ou coordenação da vigilância em saúde; planejamento em saúde; educação permanente e educação em saúde; empresas e planos de saúde privados, indústria farmacêutica, entre outros.

Por fim, outro ponto importante é a proposição de estratégias que possam ampliar a inserção desses profissionais no mercado de trabalho a fim de se ampliar e consolidar o espaço de práticas dos sanitaristas bacharéis. Viana e Souza (2018) apontam em seu estudo que algumas estratégias para isso são: "mobilização coletiva e também as iniciativas individuais dos estudantes e egressos, além da sensibilização dos gestores e ampla divulgação do curso e do profissional formado em SC". Com essas articulações, é possível trabalhar formas de ampliar esse reconhecimento e consolidar o escopo de atuação dos egressos dos CGSC.

\section{Considerações Finais}

Essa revisão integrativa da literatura é precursora na análise dos artigos originais, produzidos sobre os CGSC no Brasil. O estudo permitiu a sumarização das pesquisas realizadas e trouxe um panorama atual sobre o tema. A Graduação em Saúde Coletiva é um fenômeno recente no Brasil, desta forma, a produção científica sobre os CGSC ainda é incipiente, no entanto vem se ampliando ao longo dos anos.

Constatou-se que as pesquisas sobre a nova graduação têm se voltado para os aspectos relacionados à própria formação, à caracterização da identidade profissional e como vem ocorrendo a inserção do sanitarista graduado no mercado de trabalho. A discussão sobre a identidade profissional emerge tanto nas publicações que se debruçaram sobre a análise dos currículos, como também em um dos estudos sobre a inserção no mercado de trabalho. Portanto, a compreensão do perfil de formação e a forma como os egressos se inserem no mercado de trabalho, serão determinantes para compreender e delinear a identidade do sanitarista graduado. Os estudos analisados apontam para um perfil profissional orientado, especialmente, para a gestão dos serviços e sistemas de saúde. Com atuação voltada fundamentalmente para o SUS, nas diferentes esferas (municipal, estadual e federal). 
No tocante à inserção no mercado de trabalho, o que se observou nos estudos foram dificuldades relatadas por egressos relacionadas ao reconhecimento do sanitarista graduado no mercado de trabalho, disputa de mercado com sanitaristas pós-graduados, desconhecimento da graduação por parte dos gestores, baixa remuneração, cargos comissionados e temporários. Embora haja a necessidade de recursos humanos qualificados para a gestão, destaca-se que há um descompasso entre a necessidade de profissionais com esse novo perfil e a sua inserção no mercado de trabalho. Este vem sendo conquistado paralelamente ao desenvolvimento dos CGSC e requer tempo. A dificuldade de inserção e precarização dos vínculos é uma tendência que afeta não apenas o sanitarista graduado, mas outras categorias profissionais.

Esse estudo voltou-se à análise da literatura atual sobre os CGSC, o que pode ser um fator limitante para análises detalhadas dos contextos específicos de cada realidade observada, principalmente por se tratar de um número limitado de estudos, com metodologias distintas. Entretanto, pode-se destacar como um dos principais desafios a articulação entre as instituições de ensino e os serviços de saúde, como um fator imprescindível para a difusão do papel do sanitarista graduado.

Os resultados encontrados nesta revisão suscitam questões que merecem se respondidas através de novos estudos, com busca de fontes de dados primários, sejam elas documentais ou investigadas diretamente com os atores envolvidos (gestores, docentes, estudantes e egressos dos CGSC). Os PPP mostraram-se fontes ricas que permitem caracterizar a estrutura da formação e merecem ser reavaliados à medida que os cursos passam por atualizações e adequações à proposta da minuta das DCN para os CGSC. Também são necessárias pesquisas que identifiquem e caracterizem o perfil do sanitarista graduado, os cenários de práticas, de forma a investigar se há outros espaços para atuação, além da gestão. No tocante à inserção do sanitarista no mercado de trabalho, questões sobre as barreiras existentes para inserção, estratégias para melhorar o acesso ao mercado, assim como possíveis diferenças existentes entre a inserção de sanitaristas graduados e pós-graduados merecem ser investigadas.

\section{Referências}

Antunes, R. (2018). O privilégio da servidão [recurso eletrônico]: o novo proletariado de serviços na era digital. Boitempo.

Baptista, T. V. de F., Azevedo, C. S., \& Machado, C. V. (2015). Políticas, Planejamento e Gestão em Saúde: abordagens e métodos de pesquisa. Fiocruz.

Bosi, M. L. M., \& Paim, J. S. (2010). Graduação em Saúde Coletiva: limites e possibilidades como estratégia de formação profissional. Ciência \& Saúde Coletiva, 15(4), 2029-2038.

Brasil. Conselho Nacional de Saúde. (2013). Resolução ${ }^{\circ}$ 466, de 12 de dezembro de 2012. Aprova normas regulamentadoras de pesquisas envolvendo seres humanos. Diário Oficial da União.

Cezar, D. M., et al. (2015). O Bacharel em Saúde Coletiva e o mundo do trabalho: uma análise sobre editais para concursos públicos no âmbito do Sis tema Único de Saúde. Saúde em redes. 1(4), 65-73.

Faria, M. A. de, \& Silva, A. de J. (2016). Gestão de serviços de saúde: analisando a identidade na graduação. Saúde em Debate, 40(108), 95105. https://doi.org/10.1590/0103-1104-20161080008

Gerhardt, T. E. (2019). Cultura e cuidado: dilemas e desafios do ensino da antropologia na graduação em Saúde Coletiva. Saúde e Sociedade, 28(2), 38-52. https://dx.doi.org/10.1590/s0104-12902019190127

Ianni A. M. Z. (2016). Sobre como estamos todos implicados no desafio da formação em saúde pública/coletiva para o século XXI, In: Lorena, A. G; Akerman, M. Uma vou várias? Identidades para o sanitarista! Hucitec.

Loiola, A A., et al (2017). Competências e habilidades nos currículos da graduação em saúde coletiva no Brasil. Rev. Baiana de Saúde Pública. 41 (1), 81-97.

Lorena, A. G. de, et al (2016). Graduação em saúde coletiva no Brasil: onde estão atuando os egressos dessa formação? Saúde e Sociedade, 25(2), 369-380. https://doi.org/10.1590/S0104-12902016158123

Lorena, A. G. de, \& Akerman, M. (2016). Uma ou várias? Identidades para o sanitarista! Hucitec.

Mendes, K. D. S., et al (2008). Revisão integrativa: método de pesquisa para a incorporação de evidências na saúde e na enfermagem. Texto \& Contexto Enfermagem, 17(4), 758-764. https://dx.doi.org/10.1590/S0104-07072008000400018

Meneses, J. J. S., et al (2017). Panorama dos cursos de graduação em saúde coletiva no Brasil entre 2008 e 2014. Trabalho, Educação e Saúde, 15(2), 501518. https://dx.doi.org/10.1590/1981-7746-so100060 
Research, Society and Development, v. 10, n. 5, e21810514746, 2021

(CC BY 4.0) | ISSN 2525-3409 | DOI: http://dx.doi.org/10.33448/rsd-v10i5.14746

Ministério da educação (2020). Cadastro Nacional de Cursos e Instituições de Educação Superior: Cadastro e-MEC. http://emec.mec.gov.br/emec/nova\#simples.

Minuta de Diretrizes Curriculares Nacionais para os Cursos de Graduação em Saúde Coletiva. (2017). http://portal.mec.gov.br/docman/setembro-2017pdf/72431-pces242-17-pdf/file.

Nunes, Tânia Celeste Matos, Fagundes, Terezinha de Lisieux Quesado, \& Soares, Cathatina Leite Matos. (2015). Os Cursos Lato Sensu na Formação em Saúde Coletiva: evolução histórica e desafios contemporâneos. In: Lima, Nísia Trindade (Org.) Saúde Coletiva: a Abrasco em 35 anos de história. Fiocruz.

Osmo, A., \& Schraiber, L. B. (2015). O campo da Saúde Coletiva no Brasil: definições e debates em sua constituição. Saúde e Sociedade, 24(Suppl. 1), 205218. https://doi.org/10.1590/s0104-12902015s01018

Paim, J. S. (2006). Desafios para a saúde coletiva no século XXI. EDUFBA.

Paim, J. S. (2007). Reforma Sanitária Brasileira: contribuição para a compreensão e crítica. Tese (Doutorado em Saúde Coletiva) - Instituto de Saúde Coletiva, Universidade Federal da Bahia. Salvador, BA.

Paim, J. S., \& Pinto, I. C. M. (2013). Graduação em Saúde Coletiva: conquistas e passos para além do sanitarismo. Tempus: Actas Saúde Coletiva, 7(3), 13-35.

Paro, C. A., \& Pinheiro, R. (2018). Interprofissionalidade na graduação em Saúde Coletiva: olhares a partir dos cenários diversificados de aprendizagem. Interface - Comunicação, Saúde, Educação, 22(2), 1577-1588. https://doi.org/10.1590/1807-57622017.0838

Pinto, I. C. P., \& Paim, J. S. (2015). A ABRASCO e a experiência da Graduação em Saúde Coletiva. In: Lima, N. T., Santana, J. P., And Paiva, C. H. A., orgs. Saúde coletiva: a Abrasco em 35 anos de história. Fiocruz.

Pereira, M. F. (2018). Interprofissionalidade e saúde: conexões e fronteiras em transformação. Interface - Comunicação, Saúde, Educação, 22(Suppl. 2), 17531756. https://doi.org/10.1590/1807-57622018.0469

Pochmann, M. (2020). Tendências estruturais do mundo do trabalho no Brasil. Ciência \& Saúde Coletiva,25(1), 89-99. Epub December 20, 2019.https://doi.org/10.1590/1413-81232020251.29562019

Silva, V. O. da, \& Pinto, I. C. de M. (2013). Construção da identidade dos atores da Saúde Coletiva no Brasil: uma revisão da literatura. Interface Comunicação, Saúde, Educação, 17(46), 549-560. https://dx.doi.org/10.1590/S1414-32832013000300005

Silva, V. O. da, \& Pinto, I. C. de M. (2018). Identidade do sanitarista no Brasil: percepções de estudantes e egressos de cursos de graduação em Saúde Pública/Coletiva. Interface - Comunicação, Saúde, Educação, 22(65), 539-550. https://dx.doi.org/10.1590/1807-57622016.0825

Silva, V. O., et al (2018). Identidade profissional e movimentos de emprego de egressos dos cursos de graduação em Saúde Coletiva. Saúde em Debate, 42(119), 799-808. https://doi.org/10.1590/0103-1104201811901

Sobral, L. F., et al (2017). A área de política, planejamento e gestão em saúde nas graduações em saúde coletiva no Brasil. Trabalho, Educação e Saúde, 15(3), 879-894. https://dx.doi.org/10.1590/1981-7746-sol00076

Souza, P. B. (2018). Mercado de trabalho do sanitarista em saúde coletiva: novas perspectivas. Revista Baiana de Saúde Pública. $42(4)$, a2840.

Viana, J. L., \& Souza, E. C. F. (2018). Os novos sanitaristas no mundo do trabalho: um estudo com graduados em saúde coletiva. Trabalho, Educação e Saúde, 16(3), 1261-1285. https://doi.org/10.1590/1981-7746-sol00146

Viana, J. L. (2017) Sou bacharel em saúde coletiva, e agora? Sobre quando novos sanitaristas entram no mundo do trabalho. Dis sertação (Mestrado em Saúde Coletiva) - Universidade Federal do Rio Grande do Norte, Natal, RN.

Vieira-da-Silva, L. M. (2018). O Campo da Saúde Coletiva: gênese, transformações e articulações com a reforma sanitária. EDUFBA. Fiocruz. 